\title{
Prevalence of malnutrition in community meal users in Kensington and Chelsea
}

\author{
J. Taylor \\ Eastry House, Walmer Road Clinic, 226 Walmer Road, London W11 4ET, UK
}

There are few data available on the nutritional status of community meal users in the UK. A local profiling of the community meals service in Kensington and Chelsea, conducted by the Caroline Walker Trust ${ }^{(1)}$, identified a number of factors which may impact on a service users' nutritional intake. It was highlighted that community meals users were choosing menu options that were not appropriate to their current needs, experienced social isolation and had increased need for support around food. Only a limited number of users were receiving dietetic intervention. Despite the role community meal provision plays in supporting this user group, additional interventions may be required to ensure nutritional needs are met.

The Royal Borough of Kensington and Chelsea Adult Social Care Team provided contact details of all consenting community meal users. Users were visited by a registered dietitian in their home and were screened using the Malnutrition Universal Screening Tool $(\text { MUST })^{(2)}$ from March to April 2010. Referral to appropriate services was made as required.

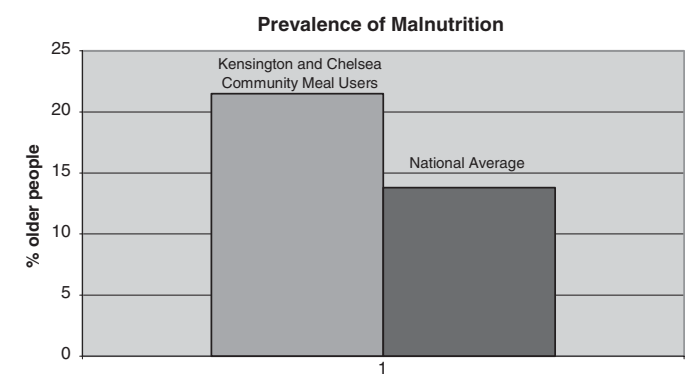

One hundred and six (mean age 83 (SD 10.03) years; $71 \%$ female) were screened for malnutrition using MUST ${ }^{(2)}$. Overall prevalence of malnutrition was $21.5 \%$ with $9.4 \%$ at high risk and $12.1 \%$ at medium risk. Twenty-one users were referred to the nutrition support team for further dietetic input, 4 users to Age Concern Food and Friends for meal companionship and 7 users to other health professionals.

The national prevalence of malnutrition in older people living in the community is $13.8 \%{ }^{(2)}$. The data collected indicate that the prevalence of malnutrition is $7.7 \%$ higher in Kensington and Chelsea. Nutritional intervention is recommended to reduce the risks associated with malnutrition in these users. The delivery of community meals involves a number of different agencies working together including meal delivery drivers, community support services and adult social care. An integrated approach has been adopted across Kensington and Chelsea in order to ensure risk of malnutrition is identified early and treated appropriately. A number of key changes have been introduced, such as mandatory referral to the Nutrition and Dietetic Service for all consenting community meal users. Malnutrition awareness training has been made available for all staff, including home care staff, social workers, support staff and community meals drivers. This is to ensure increased knowledge and confidence in the identification and treatment of malnutrition and signposting to appropriate services. Access to community-based 'meal companions' through the Age Concern Food and Friends programme has allowed assistance and support for users during mealtimes. All dietitians have access to the electronic database Nutridata, where the assessment of nutrient-based meal profiles for all community meals supplied by Apetito is available, thus ensuring that appropriate meal selection for users may be recommended. The impact of these service changes and user outcomes will be evaluated to ensure that malnutrition risk is minimised and effectively managed.

1. Caroline Walker Trust (2010) Community Meal Service Review.

2. British Association Parental and Enteral Nutrition (2003) Malnutrition Universal Screening Tool (MUST). http://www.bapen.org.uk. 